\title{
Loose regulation of medical marijuana programs associated with higher rates of adult marijuana use but not cannabis use disorder
}

\author{
Arthur Robin Williams (D), Julian Santaella-Tenorio (D), Christine M. Mauro (D), Frances R. Levin \& \\ Silvia S. Martins
}

Columbia University, New York, NY, USA

\begin{abstract}
Background and Aims Most US states have passed medical marijuana laws (MMLs), with great variation in program regulation impacting enrollment rates. We aimed to compare changes in rates of marijuana use, heavy use and cannabis use disorder across age groups while accounting for whether states enacted medicalized (highly regulated) or non-medical mml programs. Design Difference-in-differences estimates with time-varying state-level MML coded by program type (medicalized versus non-medical). Multi-level linear regression models adjusted for state-level random effects and covariates as well as historical trends in use. Setting Nation-wide cross-sectional survey data from the US National Survey of Drug Use and Health (NSDUH) restricted use data portal aggregated at the state level. Participants Participants comprised 2004-13 NSDUH respondents ( $n \sim 67$ 500/year); age groups 12-17, 18-25 and 26+ years. States had implemented eight medicalized and 15 non-medical MML programs. Measurements Primary outcome measures included (1) active (past-month) marijuana use; (2) heavy use (> 300 days/year); and (3) cannabis use disorder diagnosis, based on DSMIV criteria. Covariates included program type, age group and state-level characteristics throughout the study period. Findings Adults 26+ years of age living in states with non-medical MML programs increased past-month marijuana use $1.46 \%$ (from 4.13 to $6.59 \%, P=0.01$ ), skewing towards greater heavy marijuana by $2.36 \%$ (from 14.94 to 17.30 , $P=0.09$ ) after MMLs were enacted. However, no associated increase in the prevalence of cannabis use disorder was found during the study period. Our findings do not show increases in prevalence of marijuana use among adults in states with medicalized MML programs. Additionally, there were no increases in adolescent or young adult marijuana outcomes following MML passage, irrespective of program type. Conclusions Non-medical marijuana laws enacted in US states are associated with increased marijuana use, but only among adults aged 26+ years. Researchers and policymakers should consider program regulation and subgroup characteristics (i.e. demographics) when assessing for population level outcomes. Researchers and policymakers should consider program regulation and subgroup characteristics (i.e. demographics) when assessing for population level outcomes.
\end{abstract}

Keywords Adolescent drug use, cannabis use disorder, epidemiology, marijuana, medical marijuana laws, public health, state policy.

Correspondence to: Arthur Robin Williams, Division on Substance Use Disorders, Columbia University Department of Psychiatry, New York State Psychiatric Institute, 1051 Riverside Drive, Unit 66, New York, New York 10032, USA. E-mail: aw2879@cumc.columbia.edu Submitted 20 December 2016; initial review completed 27 February 2017; final version accepted 6 June 2017

\section{INTRODUCTION}

The United States has entered a new era of marijuana control policy during the past two decades [1]. As of the November 2016 election, 28 states and DC had legalized the use of marijuana for medical indications through the passage of medical marijuana laws (MMLs), either by voter initiative or legislative action. Additionally, eight states and Washington
DC (all of which allow for medical marijuana) have now fully legalized the recreational use of marijuana by adults aged more than 21 years, with implementation first beginning in Colorado in 2014. In addition to differing combinations of these laws, states also vary tremendously in regulations guiding their MML programs and participant eligibility [1,2].

Studies that investigate the impact of MML on rates of adolescent and adult marijuana use, heavy use and 
cannabis use disorder have produced mixed findings [3,4]. This may be due in part to imprecise categorization of states. For instance, in a recent study, Williams et al. [5] found that highly medicalized state MML programs (those requiring use of cannabinoid products with state licensed manufacturing, dispensing, testing and labeling under close physician supervision) had much lower rates of enrollment, whereas states with non-medical MML programs (i.e. loosely regulated with few restrictions) involved more than 99.4\% of the approximately 1 million MML program participants active nation-wide as of October, 2014 [5].

Although MML program regulations may impact the volume and characteristics of participants, there is also a probable time effect on the cumulative number of people participating in a given state (i.e. increasing participation over time). Because loosely regulated non-medical MML programs were passed disproportionately early (and therefore have been operating the longest) it is more challenging to separate out the impact of regulation versus time. Additionally, it is now understood widely that MML program participation was largely suppressed until 2008-09, due to early concerns about possible federal enforcement that would jeopardize patients and providers [5]. As a result, studies of MML programs would probably be more accurate if adjusting for MML passage in relation to 2008. Finally, depending upon MML program regulation and resultant patient characteristics, it may be that some states experience higher prevalence of cannabis use disorder among marijuana users. Higher prevalence of cannabis use disorder and related consequences would be of great concern, given the rapidly expanding use of marijuana nation-wide [4].

To determine whether accounting for variation in program regulation clarifies the potential impact of MMLs on prevalences of active (past-month) marijuana use, heavy use ( $>300$ days/year) and cannabis use disorder, we studied the 50 states during the period 2004-13 to assess trends in National Survey on Drug Use and Health (NSDUH) data over time with particular emphasis on age groups. Our aims were to: (1) identify whether passage of medicalized versus non-medical MML programs had a differential impact on rates of marijuana use, heavy use or cannabis use disorder and (2), according to age group (adolescents aged 12-17, young adults aged 18-25 and adults aged $26+$ ) during our study period.

\section{METHODS}

Design

In this study, we used a difference-in-differences regression to examine changes in prevalence of marijuana use, heavy use and cannabis use disorder after enactment of medicalized and non-medical MML programs, with aggregate state-level data. Supporting information, Table S1 shows the classification of the 23 states with operating programs by the end of 2014 according to whether they enacted medicalized $(n=8)$ or non-medical $(n=15)$ programs. Washington DC was excluded from all analyses.

\section{Data}

We used cross-sectional survey data from the National Survey of Drug Use and Health (NSDUH) restricted use data portal from 2004 to 2013 to obtain prevalences of marijuana use outcomes at the state level by year. NSDUH provides estimates of drug and alcohol use prevalence, as well as drug use patterns and use-related problems, in the general US civilian population aged 12 and older [6]. A multistage probability sampling design allows for computation of prevalence estimates that are representative of states and national populations. Design-based weights adjust for non-response and post-stratify to the known total US population aged 12 and older [7]. The NSDUH design also allows for computation of representative estimates for different socio-demographic groups at the state level. Each year, NSDUH surveys $\sim 67500$ individuals, with an oversample of younger age groups ( 17500 youth aged $12-17$ years and $\sim 17500$ young adults aged $18-25$ years $[7,8]$.

The reliability and validity of the NSDUH measures have been documented previously [9-11]. Professional field interviewers conduct interviews using computerassisted interviewing methods, allowing respondents highly private and confidential means of responding to sensitive questions, which putatively increases the level of honest reporting regarding illegal drug use and other sensitive behaviors [7]. Survey screening response rates ranged from 84 to $91 \%$ and interview response rates ranged from 72 to $77 \%$ during the 2004-13 period.

\section{Measures}

\section{Primary exposure variables}

Enactment of state-level MMLs was our primary exposure of interest. This variable was characterized by (1) whether states had enacted a MML in a specific year, as determined through review of state policies by legal scholars, economists and policy analysts at RAND Corporation [12] and (2) whether the enacted MML included a medicalized or non-medical program as determined on a previous classification by Williams et al. [5]. MMLs were categorized as a five-level, time-varying variable with the following levels (as indicated in Supporting information, Appendix S1): (1) before period for states with medicalized programs (before-med), (2) after period for states with medicalized programs (after-med), (3) before period for states with non-medical programs (before-non-med), (4) after period for states with non-medical programs (after-non-med) and (5) states never enacting MMLs before 2015 (no MML states). Eight states passed MML and four 
implemented medicalized programs by 2015: Connecticut, Delaware, Massachusetts and New Jersey. These states were coded as 'before-med' for the years before the enactment of the law and as 'after-med' for years after (see Supporting information, Appendix S1). For example, because Delaware enacted its MML in 2011, this state was coded as 'before-med' for years 2004-10 and as 'after' for years 2011-13. Similarly, the 15 states implementing MML with non-medical programs were coded as 'beforenon-med' and 'after-non-med' following the same procedure. States that had not enacted MML by 2015 were coded as 'never' for all years. This procedure built upon prior methodology by our group using before-after comparisons for all 50 states $[13,14]$.

\section{Active marijuana use}

Our primary outcome was prevalence of active (pastmonth) marijuana use across time at the state level. We used the imputation-revised variable provided by NSDUH. Prevalences were calculated for each state and each year from NSDUH individual data.

\section{Frequency of marijuana use in the past year}

NSDUH participants using marijuana in the past year responded to the following question: "We want to know how many days you've used marijuana or hashish during the past 12 months. What would be the easiest way for you to tell us how many days you've used it?'. Individuals could choose from three options: (1) average number of days per week, (2) average number of days per month or (3) total number of days in the past 12 months. Individuals were then asked about frequency of use according to their answer to the previous question: e.g. 'On how many days in the past 12 months did you use marijuana or hashish?'. NSDUH provides an imputation-revised variable aggregating responses to these previous questions. We dichotomized this variable as having used marijuana $<300$ days versus $>300$ days/year ('heavy use') in the past 12 months [15].

\section{Cannabis use disorder}

Participants were classified as having marijuana abuse or dependence based on DSM-IV criteria [16]. We used the NSDUH cannabis use disorder (abuse and/or dependence) imputation-revised variable. Prevalences were calculated for each state and year from NSDUH individual data.

\section{State-level covariates}

State-level control variables included the proportion of each state's population of male, white, aged 10-24 and at least high school-educated individuals in the population aged $>25$ years, the state unemployment rate and median household income. Census values from 2000 and 2010 were used for 2004-05 and 2006-13, respectively.

\section{Statistical analyses}

The prevalences of active marijuana use, heavy use and cannabis use disorder were calculated using Taylor's series linearization estimation methods to account for the complex survey design features of the NSDUH [17].

To examine whether states' implementation of medicalized or non-medical MML programs was associated with changes in marijuana outcomes, we used multilevel linear regression models [18] with state-level random intercepts to account for repeated measurements within states over time and also adjusted for state-level covariates. In addition, we used a piecewise cubic spline [19], with a knot at 2008 to control for the non-linearity of national trends in past-month marijuana use; this allowed us to control for any national trends that could have influenced the prevalence of marijuana use over time. Based on the NSDUH complex survey design, age was included as a threelevel predictor for age groups of interest (12-17, $18-25,26+$ years) interacting with the five-level MML variable to allow for differential effects of passage of MML and type of program (i.e. medicalized or non-medical) on marijuana use by age group.

The use of the five-level MML-type of program variable in these models allowed us to compare the average prevalence of past-month marijuana use in the before-MML period versus the after-MML period throughout MML states specifically with medicalized programs, while controlling for contemporaneous trends in states that never enacted MML; similarly, we were able to compare the average prevalence of marijuana use in the before-MML versus after-MML periods throughout states enacting non-medical programs.

\section{RESULTS}

Change in the prevalence of active marijuana use after the enactment of MML

Models adjusted for national trends showed no significant change in the prevalence of past-month marijuana use among adolescents or young adults (those aged 18-25) after the enactment of MML, irrespective of whether MML programs were medicalized or non-medical (see Table 1). Significant increases in active marijuana use, from 4.13 to $6.59 \%$ (1.46 prevalence points; $P=0.01$ ), were observed among those aged $26+$, but only in states passing non-medical MML programs (see Fig. 1).

\section{Change in the prevalence of heavy marijuana use after the enactment of MML}

There was a reduction in the prevalence of heavy marijuana use among adolescent active users, from 
Table 1 Before-after changes in marijuana outcomes by age and state MML program type.

Change in prevalence of active (past-month) marijuana use by age group and state MML program type

\begin{tabular}{|c|c|c|c|c|c|}
\hline & Before MML prevalence ${ }^{\mathrm{a}}$ & $\begin{array}{l}\text { After MML } \\
\text { prevalence }^{\mathrm{b}}\end{array}$ & $\begin{array}{l}\text { Estimate } \\
\text { (after versus before) }\end{array}$ & $95 \% C I$ & P-value \\
\hline \multicolumn{6}{|c|}{ Change in prevalence of active (past-month) marijuana use by age group and state MML program type } \\
\hline Age $12-17$, medicalized & 7.85 & 7.56 & -0.28 & $-1.70 ; 1.13$ & 0.70 \\
\hline Age $12-17$, non-medical & 8.64 & 9.21 & 0.57 & $-0.60 ; 1.74$ & 0.34 \\
\hline Age 18-25, medicalized & 20.88 & 21.27 & 0.74 & $-1.07 ; 1.84$ & 0.60 \\
\hline Age 18-25, non-medical & 21.26 & 21.90 & 0.65 & $-0.62 ; 1.91$ & 0.32 \\
\hline Age $26+$, medicalized & 4.35 & 4.51 & 0.15 & $-1.24 ; 1.55$ & 0.83 \\
\hline Age $26+$, non-medical & 4.13 & 6.59 & 1.46 & $0.33 ; 2.58$ & 0.01 \\
\hline \multicolumn{6}{|c|}{ Change in prevalence of heavy marijuana use (> 300 days/year) among users by age group and state MML program type } \\
\hline Age $12-17$, medicalized: after to before & 9.90 & 6.23 & -3.67 & $-7.24 ;-0.11$ & 0.04 \\
\hline Age $12-17$, non-medical: after to before & 10.04 & 9.18 & -0.85 & $-3.48 ; 1.78$ & 0.53 \\
\hline Age $18-25$, medicalized: after to before & 17.23 & 16.86 & -0.38 & $-4.12 ; 3.37$ & 0.84 \\
\hline Age $18-25$, non-medical: after to before & 17.37 & 19.14 & 1.77 & $-1.13 ; 4.68$ & 0.23 \\
\hline Age $26+$, medicalized: after to before & 12.79 & 12.82 & 0.03 & $-3.61 ; 3.68$ & 0.99 \\
\hline Age $26+$, non-medical: after to before & 14.94 & 17.30 & 2.36 & $-0.39 ; 5.11$ & 0.09 \\
\hline \multicolumn{6}{|c|}{ Change in prevalence of cannabis use disorder by age group and state MML program type } \\
\hline Age $12-17$, medicalized: after to before & 3.51 & 3.17 & -0.34 & $-1.09 ; 0.42$ & 0.38 \\
\hline Age $12-17$, non-medical: after to before & 3.83 & 4.13 & 0.30 & $-0.32 ; 0.92$ & 0.34 \\
\hline Age 18-25, medicalized: after to before & 6.21 & 6.04 & -0.18 & $-0.94 ; 0.59$ & 0.64 \\
\hline Age $18-25$, non-medical: after to before & 7.07 & 6.27 & -0.80 & $-1.45 ;-0.16$ & 0.01 \\
\hline Age $26+$, medicalized: after to before & 0.64 & 0.66 & 0.02 & $-0.66 ; 0.71$ & 0.94 \\
\hline Age $26+$, non-medical: after to before & 0.81 & 0.95 & 0.14 & $-0.37 ; 0.65$ & 0.59 \\
\hline
\end{tabular}

${ }^{a}$ Average prevalence before the enactment of medical marijuana laws (MML) (eight medicalized MML states and five non-medical states had before MML data available); baverage prevalence after the enactment of MML (four medicalized MML states and 15 non-medical states had after MML data available). $\mathrm{CI}=$ confidence interval.

9.90 to $6.23 \%(P=0.04)$, in medicalized states; however, results showed no significant changes in states with non-medical MML programs (see Table 1); nor was there a significant change in the prevalence of heavy marijuana use among users aged 18-25 after the enactment of MML, irrespective of whether states had medicalized or non-medical MML programs. Only among those aged $26+$ living in states with non-medical MML programs was there an increase, from 14.94 to $17.30 \%(2.36 \%$ annually; $P=0.09)$ in the prevalence of heavy marijuana use among active users after the enactment of MML; however, this finding was just shy of significance (see Fig. 2).

Change in the prevalence of cannabis use disorder after the enactment of MML

Our results did not show evidence of increased prevalence of cannabis use disorder (abuse and/or dependence according to DSM-IV criteria) among adolescents or adults after states enacted MML, irrespective of program type (see Table 1). There was a slight reduction in prevalence of cannabis use disorder among young adults (18-25 years), from 7.07 to $6.27 \%(-0.80$ prevalence points; $P=0.01$ ), after enactment in states with nonmedical MML programs (see Fig. 3).

Sensitivity analyses were conducted for key analyses. For instance, treating each state as a fixed effect (i.e. using dummy variables) rather than as a random effect in our modeling strategy did not change our results. Although we modeled time initially using a cubic form with a spline in 2008 (given the change in the prevalence of marijuana use starting in 2008), we additionally modeled time as a fixed effect with a dummy variable for each year, which also did not impact upon our results.

\section{DISCUSSION}

Our findings show that increases in rates of marijuana use following the passage of MML are restricted to adults aged $26+$ in states implementing non-medical MML programs. Further, the magnitude of MML program impact upon rates of marijuana use may be greatest among heavy users, leading to an annual increase in near-daily users of $2.36 \%$ (absolute percentage change); however, this trend should be monitored over time.

Our findings are consistent with prior studies finding increases in rates of marijuana use among adults (but not 

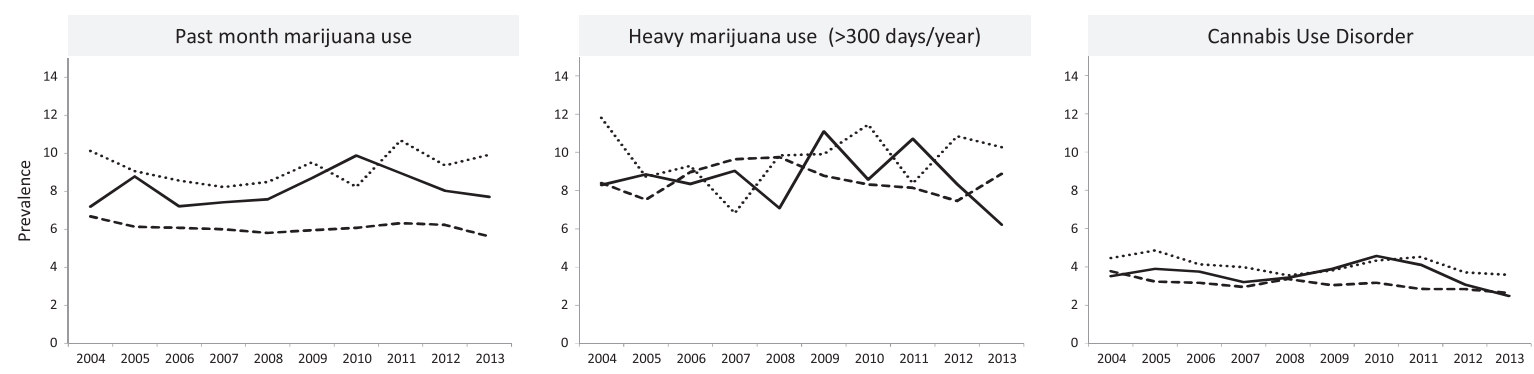

Figure I Trends in past-month marijuana use, heavy marijuana use (> 300 days/year) and marijuana use disorder among individuals aged 12-17 years. $M M L=$ medical marijuana law
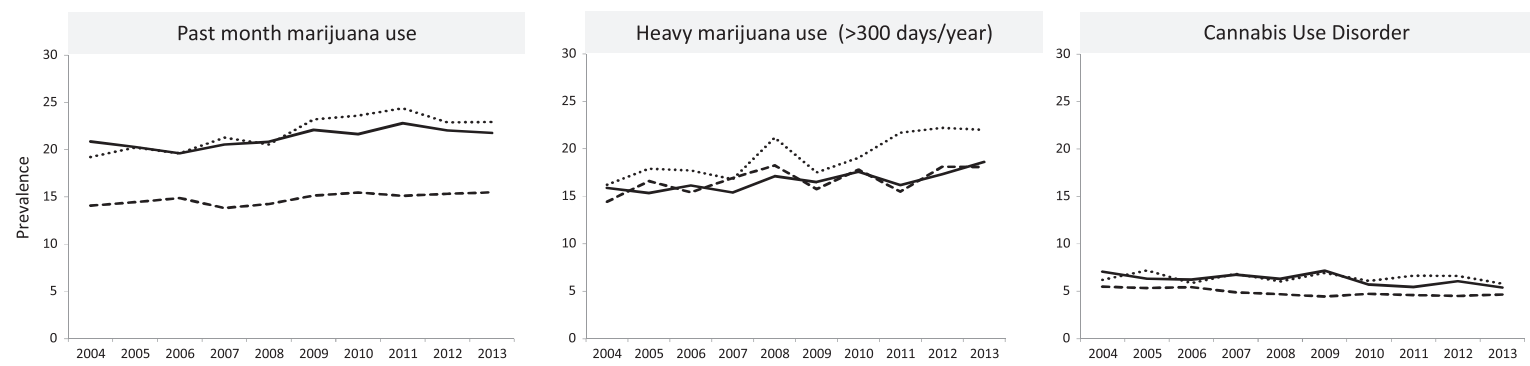

- - - States without MML by $2015 \longrightarrow$ Medicalized MML …. Non-medicalized MML

Figure 2 Trends in past-month marijuana use, heavy marjuana use (> 300 days/year) and marijuana use disorder among individuals aged 18-25 years. $\mathrm{MML}=$ medical marijuana law

adolescents) in states with MMLs. Wen and colleagues found increases in marijuana use among individuals aged more than 21 years of age in states with MMLs [3]. Martins and colleagues also found increases among adults aged 26+ in states with MMLs compared to states without MMLs [14]. Most recently, Hasin et al. 2017 [20] also found increases among adults in states with MML but did not account for variation across states in program regulation. Our results suggest that these increases may be concentrated disproportionately in states with nonmedical MML programs. As a result, the diluted effect sizes of MMLs on prevalence of marijuana use found in prior studies may betray the greater increases specific to individuals with access to loosely regulated programs with minimal physician involvement in a subset of states that implemented non-medical MML programs $(n=15$ as of 2014). However, given that only four states enacted medicalized MML programs during our study period, we may have been underpowered to detect significant changes in these states.

There is concern that with the increase in prevalence of marijuana use, especially heavy use, there will be a proportionate increase in the percentage of the population meeting diagnostic criteria for cannabis use disorder [4]. If this is the case, states with non-medical programs may bear the brunt of this increase, especially among adults aged more than 25 years. However, the increase in heavy marijuana use may, alternatively, reflect daily use for medical indications under the aegis of MMLs rather than recreational purposes [14,21].
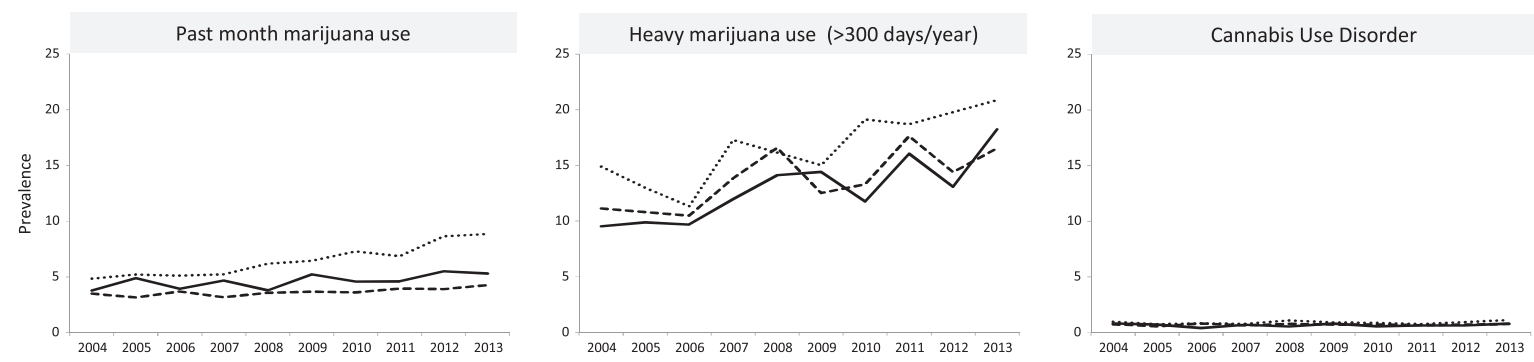

-_. States without MML by 2015 Medicalized MML ..... Non-medicalized MML

Figure 3 Trends in past-month marijuana use, heavy marijuana use (> 300 days/year) and marijuana use disorder among individuals aged $>26$ years. $M M L=$ medical marijuana law 
Although we found a slight significant reduction $(-0.80$ prevalence points, $P=0.01)$ in cannabis use disorder among young adults in states with non-medical programs, no other statistically significant changes were found. It may be too early to detect trends across states regarding the prevalence of cannabis use disorder, as the time lag between initiation of marijuana use, escalation of use and meeting diagnostic criteria for cannabis use disorder is often several years. As a result, downstream effects of changes in marijuana use prevalences may not be reflected in currently available NSDUH data on cannabis use disorder rates and might be reflected only with analyses conducted in future years with more data points. Given the tremendous interest in the potential impact of MMLs on population-level outcomes, such as rates of cannabis use disorder, and given that several of these states first passed laws almost 20 years ago, we thought it was prudent to assess for potential increases in the rates of cannabis use disorder by age category. We believe our current (largely negative) findings are relevant; however, we cannot rule out impending increases in cannabis use disorder definitively without further study.

There are several limitations to our study. Foremost, given the trend among states to pass increasingly regulated medical marijuana programs, the majority of highly medicalized programs were passed starting in 2009, limiting sample size among the newer, medicalized programs within our study period. Additionally, working with aggregated data at state-level limits our ability to assess for subgroup differences (i.e. race, sex, level of education, etc.) at the individual person-level. Further studies are needed to determine whether these findings are consistent across all subgroups of adults and adolescents, as the impact of regulation may affect individuals differentially based on gender, race or socio-economic status, in addition to age. For instance, our classification was based on a prior study by Williams et al. that relied upon seven components reflecting medicalization of program regulation. Further studies may identify alternative components (such as density of dispensaries, approved indications for use, or out of pocket costs), or combinations thereof, that have greater associations with population level outcomes. Finally, although we included several state-level covariates as random and fixed effects that may have affected our findings, there may be additional covariates impacting marijuana outcomes for which we did not control (such as other trends in substance use disorders or the burden of certain medical conditions within a given state).

\section{CONCLUSION}

Given great increases in national trends of marijuana use and heavy marijuana use among adults throughout much of the country, there is growing concern that states have been slow to address impending morbidity and social costs among the millions of Americans now using marijuana legally. Accounting for variation in state MML program regulations may help to guide prevention and treatment efforts to populations (i.e. aged 26+) within states at greater risk for unintentionally increasing rates of heavy marijuana use and related problems. Our findings suggest strongly that researchers should not treat all states with MML uniformly. Rather, key aspects of regulation that are lacking among non-medical programs, such as active physician oversight, sanctioning smoked whole plant marijuana and requiring participation in state-licensed dispensaries and the use of state-licensed products should be investigated further, as they may have a greater influence on individual morbidity and social costs than the mere passage of MMLs.

\section{Declaration of interests}

None.

\section{Acknowledgements}

Grant funding was provided through NIDA T32 DA007294-22 (Levin) and R01DA037866 (Martins).

\section{References}

1. Williams A. R. Medical and recreational marijuana policy: from prohibition to the rise of regulation. In: Compton M., editor. Marijuana and Mental Health. Arlington, VA: American Psychiatric Publishing Inc.; 2016, pp. 39-70.

2. Fairman B. J. Trends in registered medical marijuana participation across 13 US states and District of Columbia. Drug Alcohol Depend 2016; 159: 72-79.

3. Wen H., Hockenberry J. M., Cummings J. R. The effect of medical marijuana laws on adolescent and adult use of marijuana, alcohol, and other substances. J Health Econ 2015; 42: 64-80.

4. Cerdá M., Wall M., Keyes K. M., Galea S., Hasin D. Medical marijuana laws in 50 states: iInvestigating the relationship between state legalization of medical marijuana and marijuana use, abuse and dependence. Drug Alcohol Depend 2012; 120: 22-27.

5. Williams A. R., Olfson M., Kim J., Martins S. S., Kleber H. Older, less regulated medical marijuana programs have much greater enrollment rates than newer 'medicalized' programs. Health Aff 2016; 35: 480-8.

6. Substance Abuse and Mental Health Services Administration. Results from the 2013 National Survey on Drug Use and Health: Summary of National Findings, NSDUH Series H-48, HHS Publication no. (SMA) 14-4863. Rockville, MD: Substance Abuse and Mental Health Services Administration; 2014.

7. Center for Behavioral Health Statistics and Quality. $2013 \mathrm{Na-}$ tional Survey on Drug Use and Health: Methodological Resource Book, Results from the 2011 National Survey on Drug Use and Health: Summary of National Findings, NSDUH Series H-44, HHS Publication no. (SMA) 12-4713, Substance Abuse and Mental Health Services Administration, Rockville, MD. Rockville, MD: Substance Abuse and Mental Health Services Administration; 2015. 
8. SAMHSA. Results from the 2011 National Survey on Drug Use and Health: Summary of National Findings, NSDUH Series H-44, HHS Publication No. (SMA) 12-4713. Rockville, MD: Substance Abuse and Mental Health Services Administration; 2012.

9. Harrison L., Martin S., Enev T., Harrington D. Comparing drug testing and self-report of drug use among youths and young adults in the general population (DHHS Publication no. SMA 07-4249, methodology series M-7). Substance Abuse and Mental Health Services Administration, Office of Applied Studies. Rockville, MD: Substance Abuse and Mental Health Services Administration; 2007.

10. Hunter S., Feder M., Granger B., Piper L., Chromy J. Reliability study pretest analysis. In: Section 18, National Survey on Drug Use and Health: Methodological Resource Book, Prepared for the Substance Abuse and Mental Health Services Administration, Office of Applied Studies, under Contract no. 283-200400022, RTI/0209009. Research Triangle Park, NC: RTI International; 2005.

11. Piper L., Meyer M., Snodgrass J. Reliability study pretest final report. Substance Abuse and Mental Health Services Administration, Office of Applied Studies, Contract no. 283-200400022, RTI/0209009. Rockville, MD: Substance Abuse and Mental Health Services Administration; 2006.

12. Pacula R., Hunt P., Boustead A. Words can be deceiving: a review of variation among legally effective medical marijuana laws in the United States. J Drug Policy Anal 2014; 7: 1-19.

13. Wall M. M., Mauro C., Hasin D. S., Keyes K. M., Cerda M., Martins S. S. et al. Prevalence of marijuana use dose not differentially increase among youth after states pass medical marijuana laws: commentary on Stolzenberg et al. (2015) and reanalysis of US National Survey on drug use in households data 2002-2011. Int J Drug Policy 2016; 29: 9-13.

14. Martins S. S., Mauro C., Santaella-Tenorio J., Kim J., Cerda M., Keyes K. et al. State-level medical marijuana laws, marijuana use and perceived availability of marijuana among the general U.S. population. Drug Alcohol Depend 2016; 169: 26-32.
15. Pacek L. R., Mauro P. M., Martins S. S. Perceived risk of regular cannabis use in the United States from 2002 to 2012: differences by sex, age, and race/ethnicity. Drug Alcohol Depend 2015; 149: 232-44.

16. American Psychiatric Association (APA). Diagnostic and Statistical Manual of Mental Disorders: DSM-IV-TR. Washington, DC: American Psychiatric Association; 2000.

17. Chromy J., Abeyasekera S. Statistical analysis of survey data. In: United Nations, editor. Household Sample Surveys in Developing and Transition Countries. Studies in Methods. New York, NY: Department of Economic and Social Affairs Statistics Division; 2005, pp. 389-417.

18. Diggle P. J., Heagerty P., Liang K. Y., Zeger S. L. Analysis of Longitudinal Data, 2nd edn. Oxford, UK: Oxford University Press; 2013.

19. de Boor C. A Practical Guide to Splines. New York: SpringerVerlag; 1978.

20. Hasin D. S., Sarvet A. L., Cerda M., Keyes K. M., Stohl M., Galea S. et al. US adult illicit cannabis use, cannabis use disorder, and medical marijuana laws 1991-1992 to 2012-2013. JAMA Psychiatry 2017; 74: 579-88.

21. Compton W. M., Han B., Hughes A., Jones C. M., Blanco C. Use of marijuana for medical purposes among adults in the United States. JAMA 2017; 317: 209-11.

\section{Supporting Information}

Additional Supporting Information may be found online in the supporting information tab for this article.

Appendix S1 State MML Program Type and Change in Prevalence of Marijuana Before and After Passage.

Table S1 State MML program type and change in prevalence of marijuana before and after passage. 